\title{
The adaptive assessment method for different energy storage applications in large-scale re-electrical load access regions
}

\author{
Zhicheng $\mathrm{Xu}^{*}$, Jun Liu, and Zhuonan Li \\ State Grid Energy Research Institute CO., Ltd., Beijing, 102209, China
}

\begin{abstract}
In the context of energy transformation, re-electrification has become an important way to build a clean and low-carbon energy system. The large-scale re-electrical load access further increases the flexibility requirements on the user demand side. The application of the energy storage system (ESS) can not only improve the degree of electrification of the energy system but also improve the energy utilization efficiency. This paper analyzes the different development modes and key characteristics of energy storage on the power supply side, grid side and demand side in large-scale re-electrical load access areas. Five dimensions (such as storage security, technology maturity, system cost, storage scenario suitability and storage scalability) are selected to characterize the feasibility of a certain energy storage technology in a given application scenario. For different application scenarios, studying the appropriate development mode and its adaptability to the environment will help the healthy and sustainable development of energy storage.
\end{abstract}

\section{Introduction}

Energy transformation is a socialized system engineering. Its fundamental task is to build a clean, low-carbon, safe and efficient new energy system [1]. The important way is to "re-electrify". As the penetration of renewable energy continues to increase, a large-scale reelectrification load is connected to the energy system. At this time, the versatile load demand behavior will introduce greater uncertainty and unpredictability. In order to improve the user's energy quality, it is necessary to install energy storage devices to improve energy supply capacity and reliability [2-4].

With the advancement of various energy storage technologies and the improvement of the economy, energy storage has been widely used on the power supply side, power grid side and user side [5-9]. However, at present, the technical route selection for ESS in different application scenarios is more based on the qualitative judgment of actual experience [10-13]. Usually, the technology selection of energy storage in different application scenarios mainly depends on a single factor. Due to the lack of quantitative comprehensive evaluation index, the economics, safety, service life, etc. of energy storage are difficult to compared. In this paper, a multi-factor comprehensive evaluation method is established to analyze the feasibility of each energy storage technology in different application scenarios. A multi-factor comprehensive evaluation method is proposed. The model evaluates the feasibility of kinds of energy storage technologies from five dimensions: storage security, technology maturity, system cost, storage scenario suitability and storage scalability. This method can quickly give the ranking of the feasibility of different energy storage technologies in a given scenario, which is convenient for decision makers to choose the appropriate energy storage technology.

\section{Different energy storage applications in the energy system}

There are many kinds of energy storage and application scenarios. According to the application field classification, the application of energy storage in the power system can be divided into three major application parts: power supply side, power grid side, and demand side. It mainly includes six application scenarios such as renewable energy integration, grid auxiliary service, transmission and distribution infrastructure service, distributed and microgrid, industrial and commercial energy storage, electric vehicles.

\footnotetext{
* Corresponding author: xu-zhicheng2008@163.com
} 


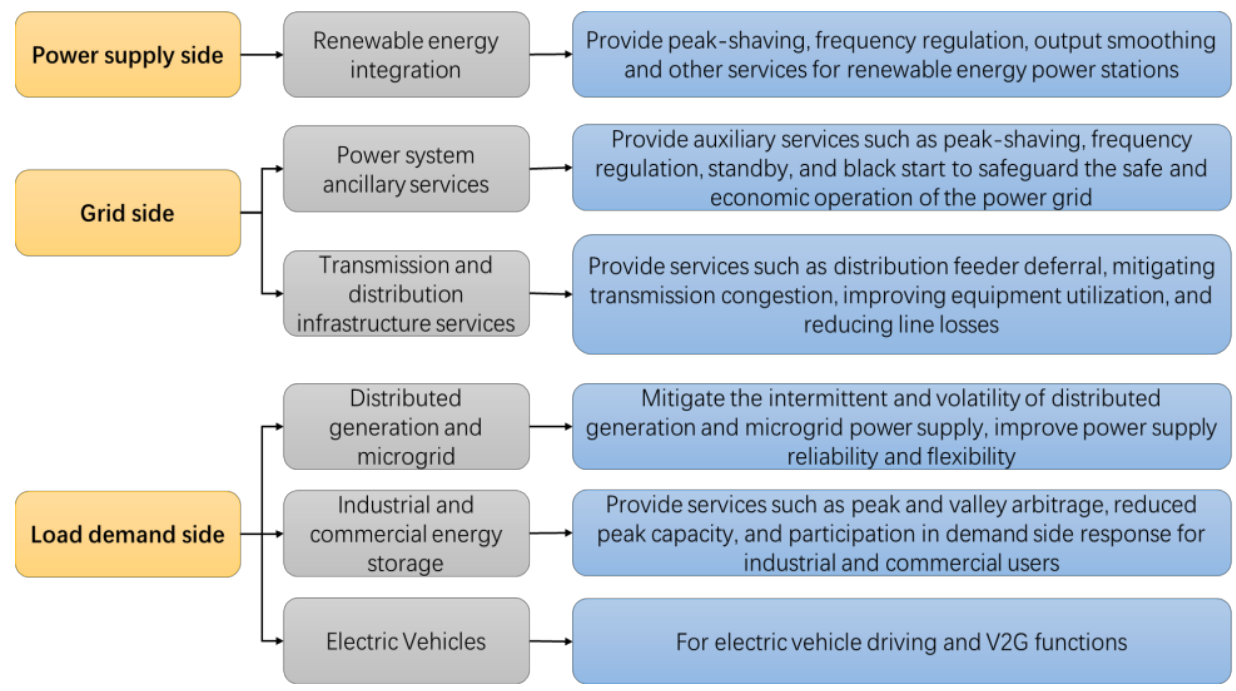

Fig. 1. Energy storage applications in different scenarios

\subsection{Energy storage applications on power supply side}

The energy storage located on power supply side mainly provides services such as smooth output, frequency modulation, reduce the rate of abandoned wind and solar power generation, and provides frequency modulation services for conventional power generators. Installing energy storage can reduce the volatility of new energy output, effectively track the output of the plan, improve the capacity of new energy consumption; improve the performance index of the power generators, reduce the cost of Automatic Generation Control (AGC) and peak regulation.

\subsection{Energy storage applications on grid side}

The energy storage located on grid side is a necessary means to cope with the balance of power systems under large-scale renewable energy integration and it is a key measure to improve grid utilization efficiency. The installation of energy storage on the grid side mainly plays the role of power grid peak shaving, new energy consumption, mitigation of congestion and improvement of overall operating efficiency of the power system and its main functions are shown as following.

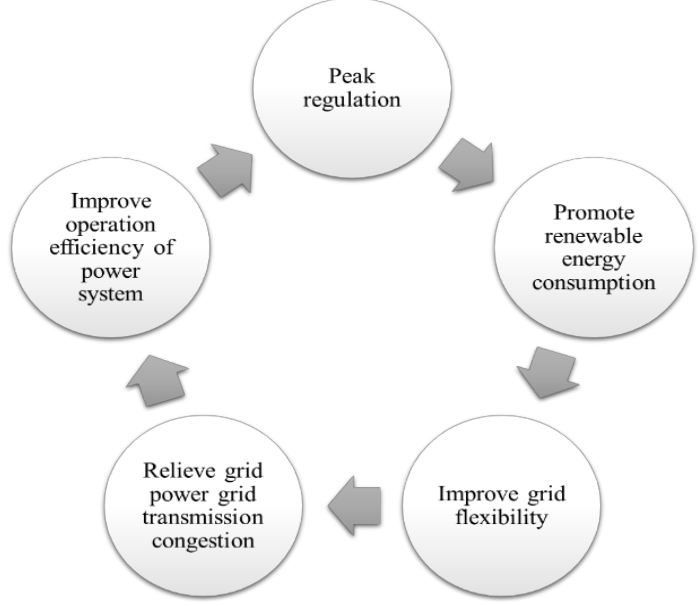

Fig. 2. Energy storage applications in the grid side

\subsection{Energy storage applications on load demand side}

The energy storage located on load demand side mainly includes microgrid energy storage, industrial and commercial energy storage, and household user energy storage. The demand side storage has the characteristics of small scale, distributed layout, and most of "uncontrollable". Reducing energy costs is an important driver for the recent development of energy storage on the user side. At present, reducing energy consumption costs through peak and valley arbitrage, reducing demand for electricity, and participating in demand response are important driving forces for user-side energy storage development.

\section{Adaptive assessment method}

At present, the selection of technical route in different application scenarios is more based on the qualitative judgment of practical experience, it is usually difficult for users to pass the field test, and compare the effect of various kinds of technical conditions under the specific scenario, so there is a lack of quantitative evaluation index. This project establishes a multi-factor comprehensive evaluation model to analyze the feasibility of each energy storage technology in different scenarios. This paper puts forward the preferred model of energy storage technology based on multi-factor comprehensive evaluation, which evaluates the feasibility of energy storage technology from five dimensions, and solves the feasible technology selection and quantification problems in the energy storage application scenario.

Firstly, based on the key feature extraction method, this paper analyzes and determines five factors such as storage security, technology maturity, system cost, storage scenario suitability and storage scalability as the key dimensions for feasibility assessment. Secondly, the weight of each key factor is determined by the expert scoring method, which makes the evaluation result more 
comprehensive and reasonable. Finally, the quantitative indicators of the feasibility of different energy storage technologies in different scenarios are calculated respectively, and the feasibility ranking is given to provide a solution for selecting suitable energy storage technologies for a given application scenario.

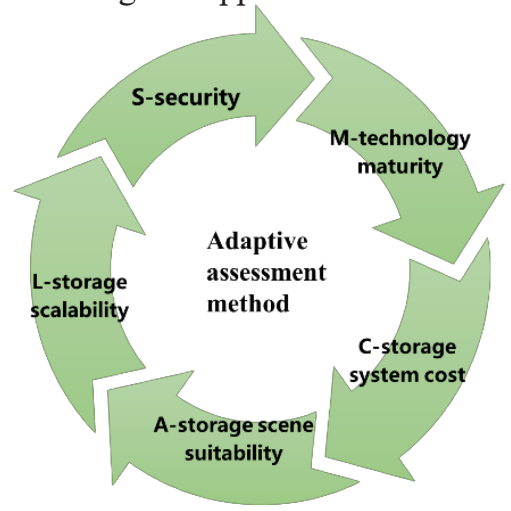

Fig. 3. A multi-factor comprehensive evaluation method for ESS

Calculate the comprehensive technical feasibility of energy storage in five dimensions according to the following formula.

ESS feasibility $=\left(S_{1}^{\alpha_{1}} \times S_{2}{ }^{\alpha_{2}} \times S_{3}^{\alpha_{3}} \times S_{4}^{\alpha_{4}} \times S_{5}^{\alpha_{5}}\right)^{\left[1 /\left(\alpha_{1}+\alpha_{2}+\alpha_{3}+\alpha_{4}+\alpha_{5}\right)\right]}$

In the formula, $S_{1}, S_{2}, S_{3}, S_{4}$, and $S_{5}$ are respectively the feasibility of various energy storage technologies

Table 1. Feasibility parameter for each energy storage technology in industrial and commercial application scenario

\begin{tabular}{|c|c|c|c|}
\hline Evaluation index / technology type & Lead carbon battery & lithium battery & Vanadium redox flow battery \\
\hline S-security & 0.85 & 0.8 & 0.95 \\
\hline M-technology maturity & 0.92 & 0.7 & 0.65 \\
\hline C-storage system cost & 0.95 & 0.8 & 0.48 \\
\hline A-storage scene suitability & 0.8 & 0.6 & 0.75 \\
\hline L-storage scalability & 1.0 & 0.8 & 0.65 \\
\hline
\end{tabular}

According to the model, the feasibility of each type of energy storage technology under different dimensions

of the application scenario is calculated and analyzed as follows.

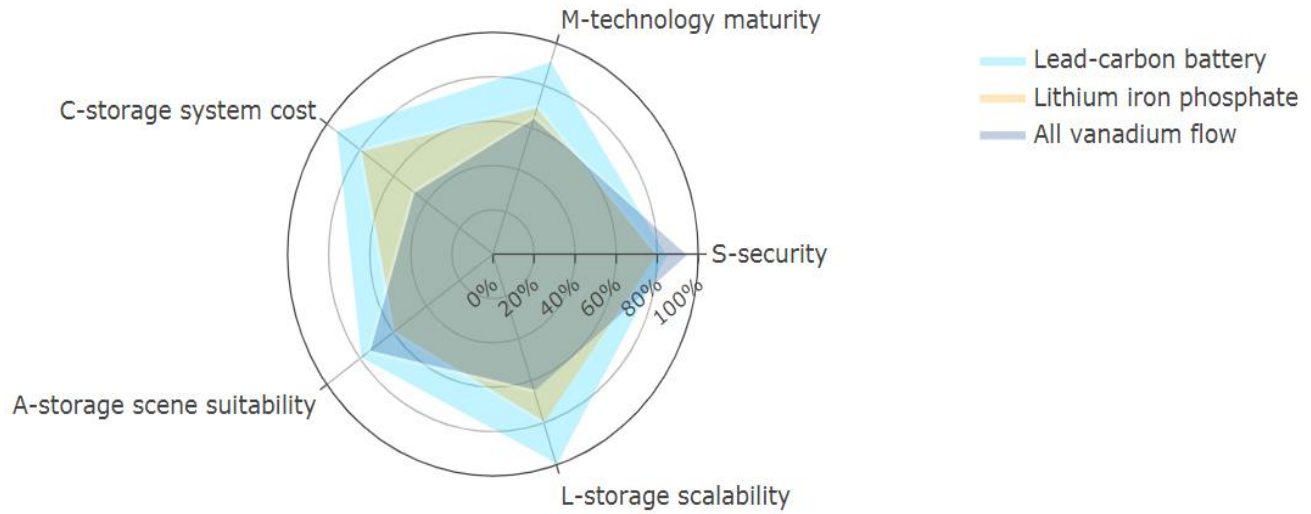

Fig. 4. Comparison and selection of radar charts of energy storage technologies in industrial and commercial energy storage scenarios 
The ranking of the feasibility of different energy storage technologies in this scenario is shown as following, and it can be known that the Lithium battery should be recommeded in the scenario.

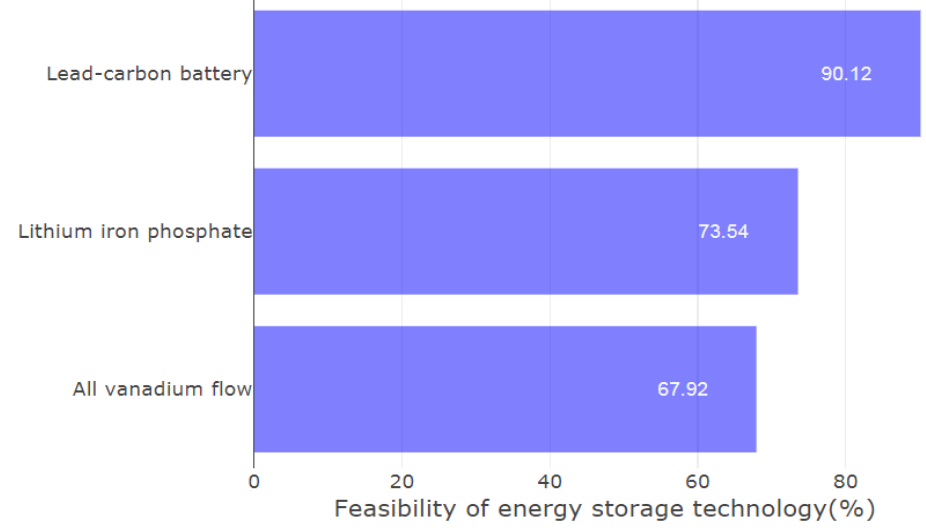

Fig. 5. Case results of energy storage technology feasibility ranking under industrial and commercial energy storage scenarios

\section{Conclusions}

This paper proposes a new comprehensive evaluation method, using a multi-factor and multi-dimensional comprehensive scoring method, to analyze the technical and economic feasibility of different energy storage technologies in a given application scenario. The results show that this method can quickly sort various technologies and give decision plans. It is worth noting that this method can be extended to the selection of energy storage technologies in any dimension.

\section{Acknowledgments}

This work was supported by the Science and Technology Project of State Grid Corporation of China (SGFJJY00GHJS1800087): Research on Technology Roadmap and Applied Policies for the Promotion of Reelectrification.

\section{References}

1. Molina M G. Energy storage and power electronics technologies: A strong combination to empower the transformation to the smart grid[J]. Proceedings of the IEEE, 2017, 105(11): 2191-2219.

2. Kear G, Shah A A, Walsh F C. Development of the all-vanadium redox flow battery for energy storage: a review of technological, financial and policy aspects[J]. International journal of energy research, 2012, 36(11): 1105-1120

3. Mahlia T M I, Saktisahdan T J, Jannifar A, et al. A review of available methods and development on energy storage; technology update[J]. Renewable and Sustainable Energy Reviews, 2014, 33: 532-545.

4. Toledo O M, Oliveira Filho D, Diniz A S A C. Distributed photovoltaic generation and energy storage systems: A review[J]. Renewable and Sustainable Energy Reviews, 2010, 14(1): 506-511.

5. Byrne C, Verbic G. Feasibility of residential battery storage for energy arbitrage[C]//2013 Australasian
Universities Power Engineering Conference (AUPEC). IEEE, 2013: 1-7.

6. Ma T, Yang H, Lu L. Feasibility study and economic analysis of pumped hydro storage and battery storage for a renewable energy powered island[J]. Energy Conversion and Management, 2014, 79: 387-397.

7. Byrne C, Verbic G. Feasibility of residential battery storage for energy arbitrage[C]//2013 Australasian Universities Power Engineering Conference (AUPEC). IEEE, 2013: 1-7.

8. Singh S, Singh M, Kaushik S C. Feasibility study of an islanded microgrid in rural area consisting of $\mathrm{PV}$, wind, biomass and battery energy storage system[J]. Energy Conversion and Management, 2016, 128: 178-190.

9. Telaretti E, Dusonchet L. Battery storage systems for peak load shaving applications: Part 2: Economic feasibility and sensitivity analysis[C]//2016 IEEE 16th International Conference on Environment and Electrical Engineering (EEEIC). IEEE, 2016: 1-6.

10. Prodromidis G N, Coutelieris F A. Simulations of economical and technical feasibility of battery and flywheel hybrid energy storage systems in autonomous projects[J]. Renewable energy, 2012, 39(1): 149-153.

11. Pearre N S, Swan L G. Technoeconomic feasibility of grid storage: Mapping electrical services and energy storage technologies[J]. Applied Energy, 2015, 137: 501-510.

12. Tang Y, Khaligh A. On the feasibility of hybrid battery/ultracapacitor energy storage systems for next generation shipboard power systems[C]//2010 IEEE Vehicle Power and Propulsion Conference. IEEE, 2010: 1-6.

13. Corey G P, Stoddard L E, Kerschen R M. Boulder city battery energy storage feasibility study[J]. Sandia Report SAND2002-0751, Sandia National Laboratories, Albuquerque, NM, 2002. 